\title{
buttonhead does not contribute to a combinatorial code proposed for
}

\section{Drosophila head development}

\author{
Ernst A. Wimmer ${ }^{1}$, Stephen M. Cohen ${ }^{2}$, Herbert Jäckle ${ }^{3}$ and Claude Desplan ${ }^{1}$ \\ ${ }^{1}$ Howard Hughes Medical Institute, The Rockefeller University, 1230 York Avenue, Box 151, New York, NY 10021-6399, USA \\ 2European Molecular Biology Laboratory, Meyerhofstrasse 1, D-69012 Heidelberg, Germany \\ ${ }^{3}$ Abteilung Molekulare Entwicklungsbiologie, Max-Planck-Institut für biophysikalische Chemie, Am Fassberg, D-37077 Göttingen, \\ Germany
}

\section{SUMMARY}

The Drosophila gap-like segmentation genes orthodenticle, empty spiracles and buttonhead (btd) are expressed and required in overlapping domains in the head region of the blastoderm stage embryo. Their expression domains correspond to two or three segment anlagen that fail to develop in each mutant. It has been proposed that these overlapping expression domains mediate head metamerization and could generate a combinatorial code to specify segment identity. To test this model, we developed a system for targeted gene expression in the early embryo, based on region specific promoters and the $f l p$-out system. Misexpression of $b t d$ in the anterior half of the blastoderm embryo directed by the hunchback proximal promoter rescues the btd mutant head phenotype to wild-type. This indicates that, while btd activity is required for the formation of specific head segments, its ectopic expression does not disturb head development. We conclude that the spatial limits of $b t d$ expression are not instructive for metamerization of the head region and that btd activity does not contribute to a combinatorial code for specification of segment identity.

Key words: buttonhead, combinatorial code, ectopic expression, empty spiracles, head development, orthodenticle, segmentation

\section{INTRODUCTION}

The Drosophila embryo has provided an excellent system for analyzing the formation of the metameric body pattern (Nüsslein-Volhard and Wieschaus, 1980). Most studies have focused on the segmentation of the trunk. They have led to the definition of an elaborate cascade of gene interactions (reviewed by Ingham, 1988). Maternally deposited determinants specify the domains of gap gene expression along the anteroposterior axis (reviewed by St Johnston and NüssleinVolhard, 1992). Gap genes then activate pair rule genes in repetitive patterns (reviewed by Pankratz and Jäckle, 1993). These, in turn, define the metameric expression patterns of segment polarity genes (reviewed by Martinez-Arias, 1993). In addition, the gap genes together with the pair rule genes define the spatial domains of the homeotic selector genes which assign segment identity (reviewed by Akam, 1987; McGinnis and Krumlauf, 1992).

Head and trunk segmentation differ in several respects. The gap genes acting in the trunk region, such as hunchback $(h b)$, Krüppel or knirps, are expressed in adjacent domains with relatively small overlaps (reviewed by Pankratz and Jäckle, 1993). The corresponding expression domains of the head gap-like segmentation genes orthodenticle (otd), empty spiracles (ems) and btd are widely overlapping (Dalton et al., 1989; Finkelstein and Perrimon, 1990; Walldorf and Gehring, 1992; Wimmer et al., 1993). Whereas the gap genes in the trunk region regulate each other's expression (reviewed by Pankratz and Jäckle, 1993), no cross-regulation between the head gap genes has been observed (Cohen and Jürgens, 1990; Wimmer et al., 1995; Gao et al., 1996).

Phenotypic analyses of mutations in the head gap genes indicate that they are required for the establishment of anterior head segments (Cohen and Jürgens, 1990; Schmidt-Ott et al., 1994, 1995). These segments do not depend on the activity of pair rule genes (Macdonald et al., 1986; Cohen and Jürgens, 1990; Lardelli and Ish-Horowicz, 1993). Therefore, a mechanism different from the one described for the trunk has to be proposed to account for segmentation of the anterior head region (Cohen and Jürgens, 1990). The three head gap genes otd, ems and btd are required in overlapping domains with their posterior margins out of phase by one segment. The phasing of the deletions in the head gap gene mutants suggests that these genes are responsible for metamerization in the developing head (Cohen and Jürgens, 1990). Moreover, no known homeotic selector genes are active in the anterior embryonic head (reviewed by Cohen and Jürgens, 1991; McGinnis and Krumlauf, 1992; Jürgens and Hartenstein, 1993). Consequently, it has been proposed that the overlapping domains of head gap gene activity might also specify the segment identity of the metameres (Cohen and Jürgens, 1990).

This combinatorial model predicts that misexpression of these genes in the early blastoderm stage embryo will interfere with metamerization and alter the identity of head segments. To test this model, we generated an inducible system which places the gene of interest under the direct control of a region 
specific promoter. Since such constructs are expected to be dominant embryonic lethal, a ' $f p$-out cassette' (Struhl and Basler, 1993) was inserted to prevent the transgene from being expressed. Excision of the $f l p$-out cassette, which occurs in the male germ line, renders the fusion transgene active. Using this system, we show that ectopic expression of btd in a broad anterior domain provides functional btd activity, but does not disturb head development. The spatial limits of $b t d$ expression are therefore not informative for metamerization of the anterior head region. Moreover, according to the combinatorial code, ectopic expression of btd anterior to its normal expression domain should transform the ocular into an antennal segment anlage. Since the ectopic expression of btd does not affect the ocular segment, we conclude that btd does not contribute to a combinatorial code for head segment specification.

\section{MATERIALS AND METHODS}

\section{General flp-out cassette constructs}

The $>y^{+}>f l p$-out cassette was isolated from Act5C $>y^{+}>w g$ (pKB411; Struhl and Basler, 1993) as an NheI-fragment and cloned into pSL1180ARVRI, a derivative of pSL1180 (Pharmacia) in which the polylinker region between the EcoRV and EcoRI sites had been removed. The yellow (y) gene was removed by a SalI cut and religation to generate $\mathrm{pSL} \gg>$, which was opened by a partial NotI cut and blunted by Klenow polymerase to insert the AUG- $\beta$ gl $(\mathrm{AB})$ gene from pCHAB $\Delta$ Sal (Wimmer et al., 1993, 1995) as a blunted $3.6 \mathrm{~kb} \mathrm{XbaI}$ fragment. From the resulting construct $\mathrm{pSL}>\mathrm{AB}>$, the new $6.1 \mathrm{~kb} f p$ out cassette was isolated by an SpeI and a partial NheI cut and cloned into the $\mathrm{XbaI}$ site of $\mathrm{pCHAB} \triangle \mathrm{Xba}$ and $\mathrm{pCHABA} \mathrm{X}$ ba generating the basic constructs $\mathrm{pC}>\mathrm{AB}>^{\prime}$ and $\mathrm{pC}>\mathrm{AB}>$, respectively (Fig 1). pCHAB $\triangle X b a$ and $\mathrm{pCHABN} \triangle \mathrm{X}$ ba result from $\mathrm{XbaI}$ cuts and religations of pCHAB $\triangle$ Sal and pCHABASal, which is derived from pCHAB $\Delta$ Sal by religating the cut and blunted Not site. The $6.1 \mathrm{~kb}$ $f p$-out cassette was similarly cloned into the $\mathrm{Xba \textrm {I }}$ sites of pCbtdRV2ndB $\Delta \mathrm{Xba}$ and $\mathrm{pChb}(\mathrm{P} 2 \not \mathrm{Xba}$ (see below) to generate the constructs $\mathrm{pCbtd}>\mathrm{AB}>$ and $\mathrm{pChb}(\mathrm{P} 2)>\mathrm{AB}>$, which provide expression in the btd head stripe domain or the anterior zygotic $h b$ domain, respectively, and have left single restriction sites after the $f p$-out cassette for inserting different coding regions.

\section{Transgenic fly lines}

To generate transgenic fly lines, the constructs described below were injected together with the helper plasmid p $\Delta 2-3$ (Laski et al., 1986) into embryos of fly strain $\operatorname{Df}(1) w^{67 \mathrm{c} 23}, y$ as reported by Rubin and Spradling (1982).

\section{$b t d>A B>b t d$}

To generate pKSbtd covering all of the btd coding region, we fused in pBluescriptKS (Stratagene) the genomic $676 \mathrm{bp} \mathrm{BamHI-PstI}$ fragment, which contains upstream untranslated sequences, translation start site and encodes the first 209 amino acids (Wimmer et al., 1993), with a 1950 bp cDNA fragment, which was obtained by a partial PstI and complete NotI cut and encodes the rest of the btd protein (Wimmer et al., 1993). From pKSbtd we isolated the btd coding sequence by an EcoRV and $S s p \mathrm{I}$ cut and cloned the resulting $2.2 \mathrm{~kb}$ fragment into the blunted NotI site of pSL>AB> (see above). From the resulting construct, $\mathrm{pSL}>\mathrm{AB}>\mathrm{BTD}$, the $8.3 \mathrm{~kb}$ fragment containing the $f p$-out cassette and the $b t d$ coding region was isolated by an SpeI and a partial NheI cut and cloned into the $X b a \mathrm{I}$ site of pCbtdRV-2ndB $\Delta$ Xba generating pCbtd $>$ AB $>$ BTD. pCbtdRV-

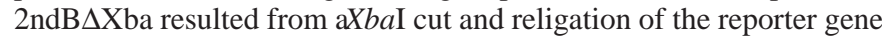
construct $b t d$ RV-2ndB (Wimmer et al., 1993, 1995). pCbtd > AB > BTD was used for P-element transformation to generate the fly strains 850 -

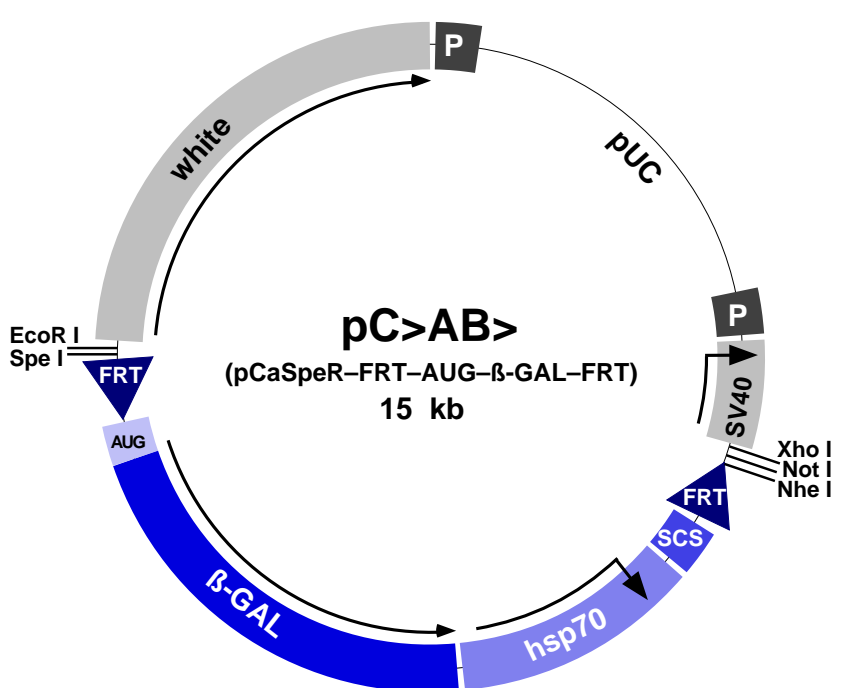

Fig. 1. Basic $f l p$-out cassette construct $\mathrm{pC}>\mathrm{AB}>$. Based on pCaSpeR (Pirrotta, 1988), this construct was designed for rapid cloning and Pelement transformation of fusion transgenes. Upstream of the $f l p$-out cassette two unique restriction sites (EcoRI, SpeI) allow the cloning of a desired promoter. Downstream of the cassette three unique cloning sites (NheI, Not I, XhoI) enable the cloning of any coding sequence of interest, which does not have to contain its own transcriptional stop signal, since the SV40 transcription stop is provided downstream of the cloning sites. The related construct $\mathrm{pC}>\mathrm{AB}>>^{\prime}$ contains an additional NotI site between the EcoRI and SpeI sites (see Materials and Methods). For details on the flp-out cassette see main text.

43 and 850-89, which carry the interrupted $b t d$ transgene $b t d>A B>b t d$ homozygous on the second chromosome.

\section{$h b>A B>b t d$}

The $h b$ proximal promoter (Schröder et al., 1988) was cloned as a 0.7 $\mathrm{kb}$ SalI-XbaI fragment (SalI site blunted) into the XbaI and blunted $E c o$ RI sites of pCHAB $\Delta \mathrm{Xba}$. Into theXbaI site of the resulting vector $\mathrm{pChb}(\mathrm{P} 2) \Delta \mathrm{Xba}$, the $8.3 \mathrm{~kb}$ fragment containing the $f p$-out cassette and the $b t d$ coding region was inserted (derived from $\mathrm{pSL}>\mathrm{AB}>\mathrm{BTD}$, see above) to generate $\mathrm{pChb}(\mathrm{P} 2)>\mathrm{AB}>\mathrm{BTD}$. This construct led, after $\mathrm{P}$ element transformation, to the isolation of five fly strains which carry the interrupted $h b>A B>b t d$ fusion transgene homozygous on the second $(857-75,857-79)$ or third chromosomes $(857-35,857-45,857$ 83).

\section{Fly strains providing flp recombinase}

For identification of embryos carrying the induced fusion transgenes (Fig. 3), we crossed the $\beta$-tubulin-flp transgene (Struhl et al., 1993) into the background of several balancer chromosomes, which were marked by different lacZ reporter gene constructs ('blue balancers'; Struhl et al., 1993). The following stocks carrying the $\beta$-tubulin- $f p$ transgene homozygous on the X-chromosome were established:

及-tub-flp;CyO hb-lacZ/cn ptc bw sp;

及-tub-flp;SM6 eve-lacZ/cn ptc bw sp;

$\beta$-tub-flp;CyO wg-lacZ/Dll ${ }^{\mathrm{B}}$;

及-tub-flp;;TM3 Sb ftz-lacZ/ru h th st cu sr e stg ca

$\beta$-tub-flp;TM3 Sb hb-lacZ/e ems ${ }^{9 \mathrm{H}}$

及-tub-flp;;TM3 Sb hb-lacZ/e ems ${ }^{9 \mathrm{Q}}$

\section{In situ hybridization and immunohistochemistry}

DNA labelling and in situ hybridization have been performed as described by Hartmann and Jäckle (1995). DNA probes were prepared 
from otd (Finkelstein et al., 1990), ems (Dalton et al., 1989) and btd (Wimmer et al., 1993) cDNAs. Antibody stainings with rabbit anti- $\beta$ galactosidase (Cappel), mouse anti-en (Patel et al., 1989), mab22C10 (Fujita et al., 1982) and rat anti-otd antibodies (Wieschaus et al., 1992) of whole-mount embryos were carried out as described by Macdonald and Struhl (1986) using the Vectastain ABC Elite horseradish peroxidase system. For double stainings, alkaline phosphatase-conjugated goat anti-rabbit antibodies (Jackson ImmunoResearch Laboratories) were used to detect rabbit anti- $\beta$-glactosidase antibodies.

\section{RESULTS}

\section{The IT-system induces targeted gene expression at blastoderm stage}

To examine the role played by the spatial domains of gap gene expression in embryonic head development, we developed a system to direct spatially restricted gene expression under the control of promoters active at early blastoderm. This system allows for immediate and targeted gene expression and will be referred to as the IT-system. Heat shock misexpression tends to cause phenocopies when applied in early embryos (Walter et al., 1990). The GAL4 system (Brand and Perrimon, 1993) cannot be used early in embryogenesis because of the lag in activation. The ideal system would be one in which a region-specific promoter is directly linked to the gene of interest. This arrangement, however, may interfere with normal development and cause dominant lethality. In order to avoid isolating only fly strains that carry low expressing or partially suppressed fusion transgenes, it is necessary to control transgene expression in an inducible manner (Parkhurst and Ish-Horowicz, 1991). To provide this function, we made use of the $f p$-out system developed by Struhl and Basler (1993) that allows the temporary separation of a region specific promoter from the coding region of a gene. We modified the $f p$-out cassette (Fig. 1) which is flanked by direct repeats of $f p$ recombinase target sites (FRTs) and inserted it between promoter and coding region. Thus, induction of $f p$ recombinase allows removal of the $f p$-out cassette and the desired fusion transgene is created.

To initially prevent expression of the coding region of interest, the $f p$-out cassette contains a transcriptional stop signal (hsp70) and a special chromatin structure (scs) element (Vasquez and Schedl, 1994) providing enhancer blocking activity (Fig. 1). To mark for the presence of the $f p$-out cassette, we inserted a lacZ gene ( $\beta$-gal; Fig. 1). ThelacZ gene serves also as a reporter gene that monitors the efficiency of the region-specific enhancer. This allows the selection of the transgenic fly lines for the ectopic expression experiments (Fig. 2F-H). To facilitate rapid cloning and germline transformation of different fusion genes, we generated the basic P-element transformation vector $\mathrm{pC}>\mathrm{AB}>$ (Fig. 1). To efficiently remove the $f p$-out cassette and activate the fusion transgene, we used fly strains carrying the $f p$ recombinase gene under the control of the $\beta$-tubulin promoter (Struhl et al., 1993). This promoter functions exclusively in maturing spermatocytes (Michiels et al., 1989). Male flies carrying both the ectopic expression construct with the $f p$-out cassette and the $\beta$-tubulin- $f p$ gene will transfer to their progeny the induced fusion transgene (Fig. 3 ). The direct control of the region specific promoter then allows expression of the fusion transgene without delay.

The intact btd transgene has been shown to rescue the btd mutant head phenotype (Wimmer et al., 1996). To test the
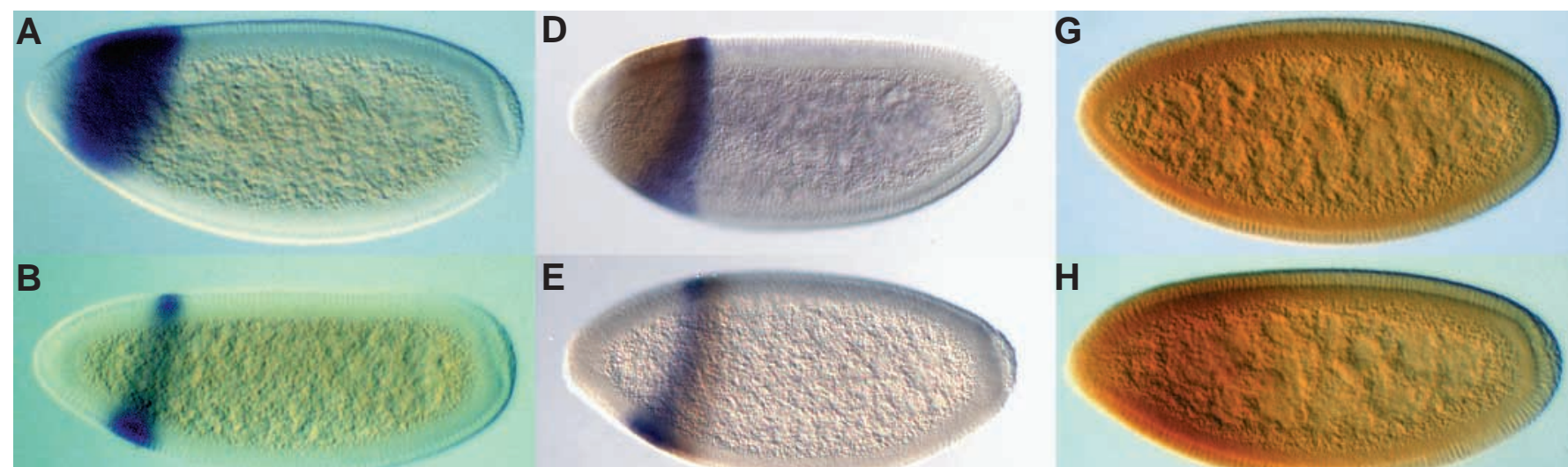

E

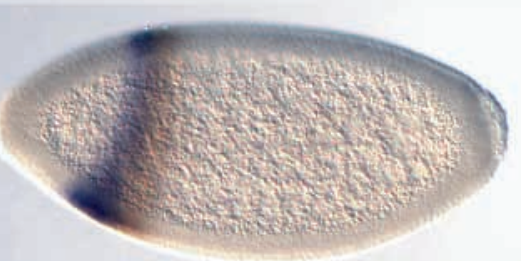

H
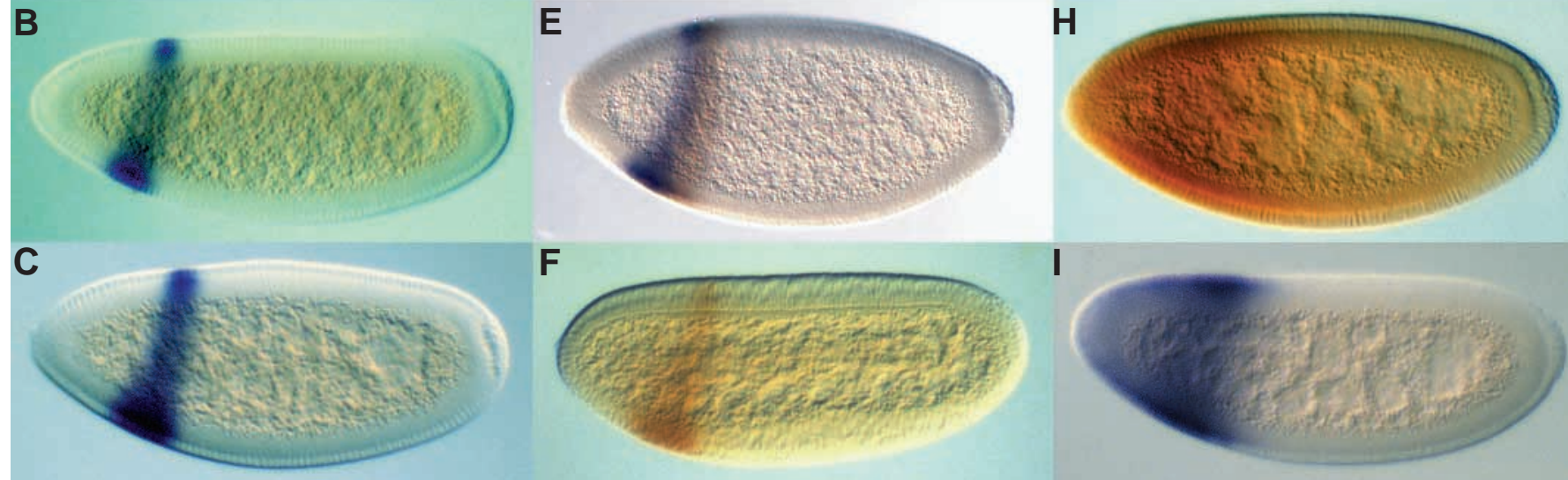

1

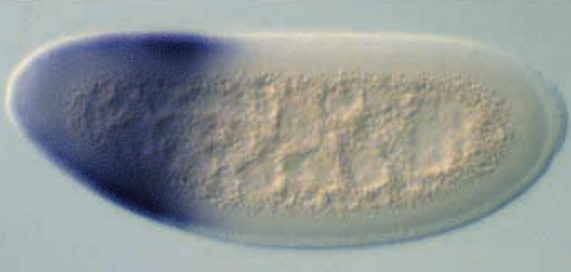

Fig. 2. Expression of head gap genes. (A) otd, (B) ems, and (C) btd mRNA expression in the syncitial blastoderm embryo. (D) Double labelings with otd (brown) and ems (blue), or (E) ems (blue) and btd (brown) DNA probes demonstrate the regions in which different combinations of head gap genes are active (Fig. 5). (F-H) Antibody staining to detect $\beta$-gl expression mediated by the uninduced transgenes $b t d>A B>b t d(\mathrm{~F})$ and $h b>A B>b t d(\mathrm{G}, \mathrm{H})$. Note that the transgenic fly lines 857-45 (G) and 857-75 (H) mediate different levels of transgene expression. (I) $b t d$ mRNA expression after $f p$-out and activation of the fusion transgene $h b>b t d$ : ectopic $b t d$ expression in the anterior half of the embryo can be detected in addition to the endogenous btd expression pattern (C). All embryos are depicted dorsal up, anterior left. 
Fig. 3. Immediate and targeted ectopic expression (IT) system. Male flies containing a $f p$-out transgene on the second or third chromosomes are crossed to virgin female flies carrying the $\beta$ tubulin-flp transgene (Struhl et al., 1993) homozygous on the $\mathrm{X}$-chromosome and a blue balancer chromosome corresponding to the $f l p$-out transgene location. Male progeny of this cross carrying the $f p$-out transgene over the blue balancer are crossed to wild type or $\mathrm{X}$-chromosomal mutant (e.g. btd) virgin females. During spermatogenesis the $f p$ recombinase induces the fusion transgene by removing the FRT-flanked $f p$-out cassette which is marked with a lac Z ( $\beta$-gl) gene. In the progeny of this cross, the coding sequence in question (gene $X$ ) will then be immediately expressed under the direct control of the region-specific promoter. Due to the marking of the $f p$-out cassette and the use of blue balancer chromosomes, embryos carrying the induced fusion transgene can be identified by their lack of $\beta$-gl expression.

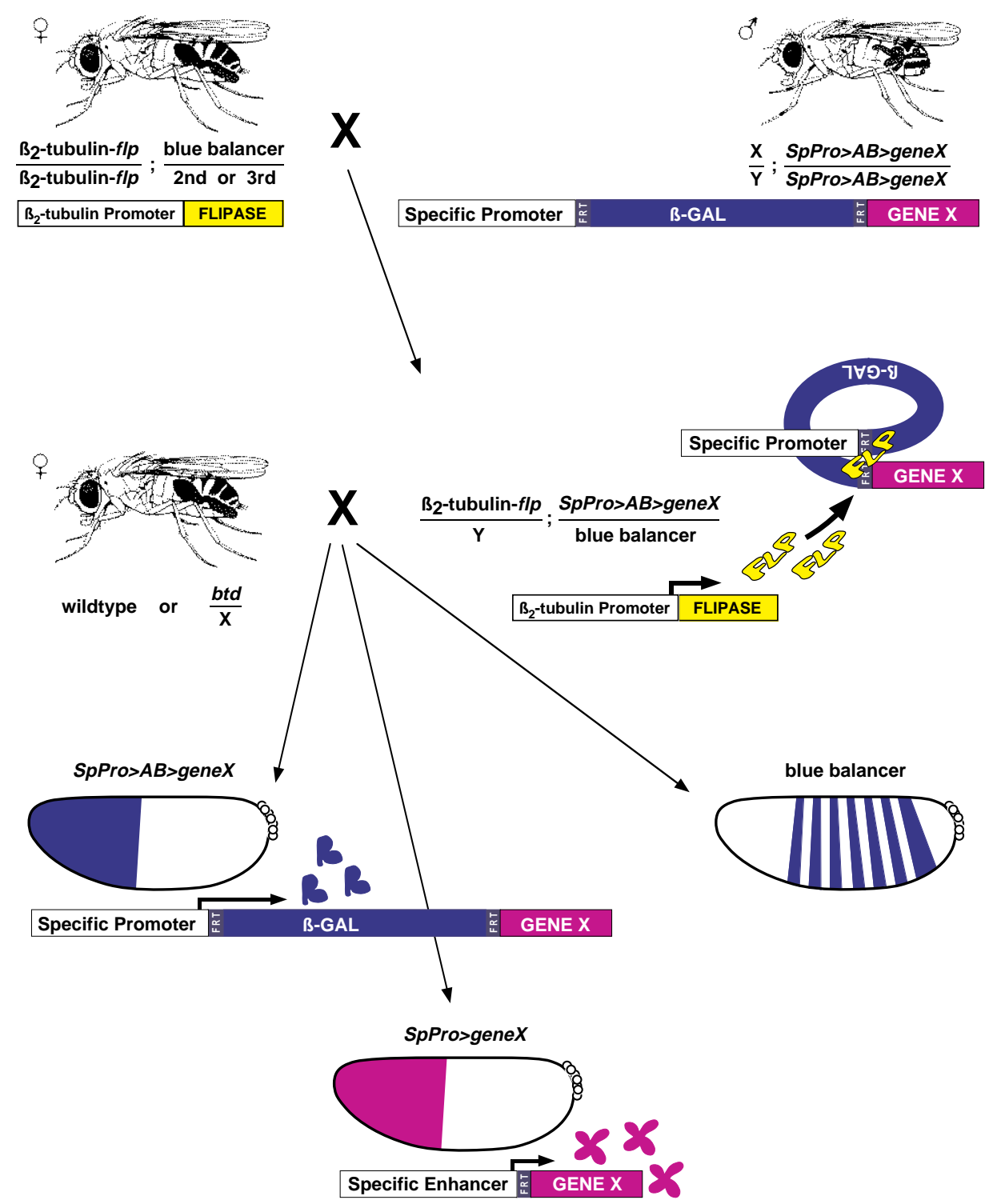

region (Cohen and Jürgens, 1990). This idea can be tested by expanding the expression domain of one of these genes, while keeping the others constant. To ask whether the spatial limits of $b t d$ expression are instructive for head metamerization, we expanded its expression domain by placing the btd coding sequence under control of the well defined proximal $h b$ promoter (Schröder et al., 1988; Driever et al., 1989; Struhl et al., 1989). We selected several transgenic fly lines, which all carry the $h b>A B>b t d$ transgene, but show different levels of lac $Z$ expression in the anterior half of the blastoderm embryo (Fig. 2G,H). Excision of the flp-out cassette allowed btd expression anteriorly and posteriorly to its normal expression domain (Fig. 2I). The ectopic $b t d$ expression is detectable until the late blastoderm stage. There are no indications that the expression mediated by the $h b$ promoter is suppressed prior to its normal fading, as had been observed in an ectopic expression study of pair-rule genes (Parkhurst and IshHorowicz, 1991). Embryos carrying the induced $h b>b t d$ fusion transgene were identified by the absence of $\beta$-gal expression

(Fig. 3).

\section{Ectopic expression of buttonhead does not affect head metamerization}

The expression patterns of the head gap genes otd, ems and btd (Fig. 2A-E) are consistent with the idea that their spatial limits have a direct input into metamerization of the anterior head 


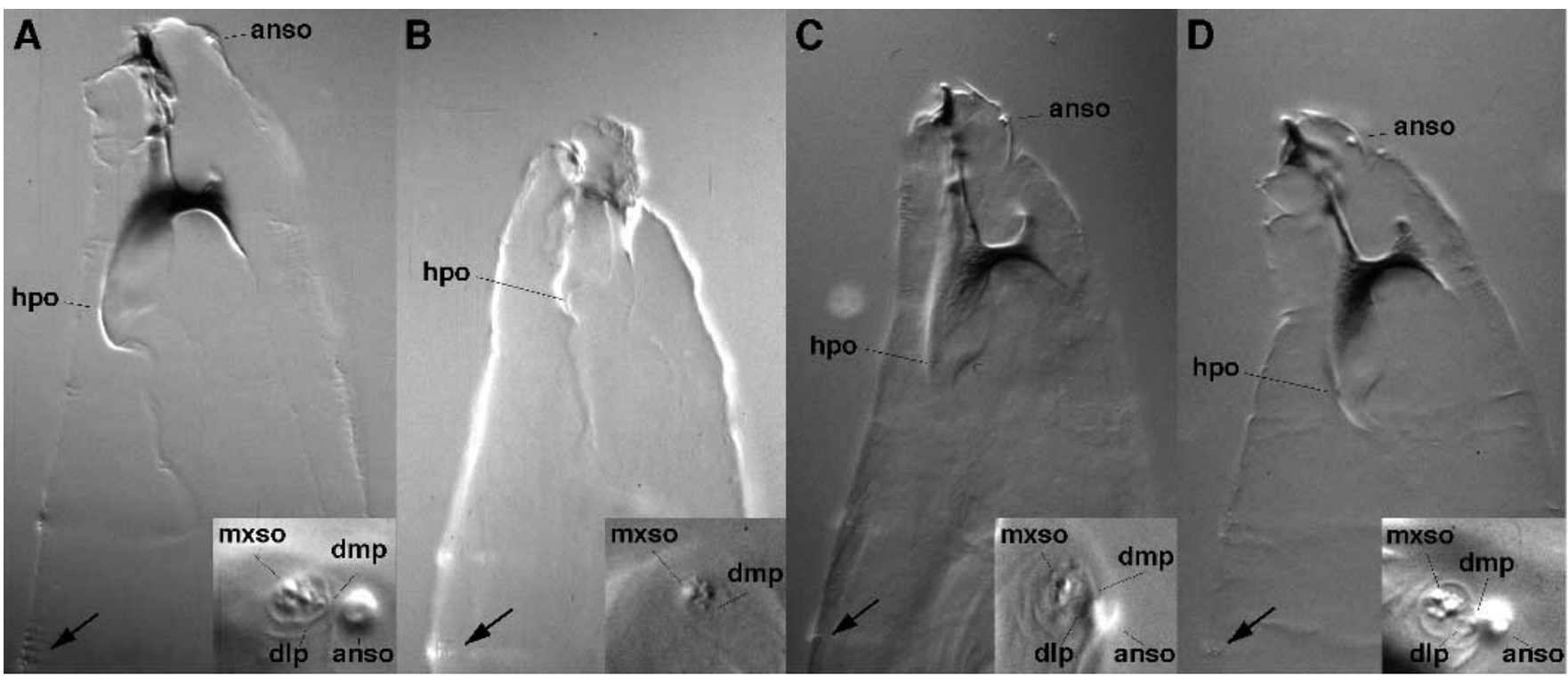

Fig. 4. Cuticle preparations of transgene rescued btd mutant embryos. (A) Wild-type cuticle. (B) Cuticle of a shaven baby (svb) btd double mutant (Wimmer et al., 1996) carrying the $f p$-out cassette containing transgene $b t d>A B>b t d$ which does not provide $b t d$ function. The $s v b$ mutation causes reduced denticle belts (arrows), whereas the $b t d$ mutation results in head deformations with the lack of antennal sense organs (anso) and dorsolateral papillae (dlp). Structures derived fom the ocular segment like the dorsomedial papillae (dmp) and the hypopharyngeal organs (hpo), as well as posterior maxillary structures like the maxillary sense organ (mxso) are not $b t d$-dependent. (C) After removal of the $f l p$ out cassette, the $b t d>b t d$ trangene provides $b t d$ function and rescues the $b t d$ head phenotype. (D) The $h b>b t d$ fusion transgene also rescues the $b t d$ mutant head phenotype. Note that structures derived from the ocular (dmp, hpo) and maxillary segments (mxso) are unaltered. The reduced denticle belts (arrows in B-D) indicate the hemizygous presence of the double mutant $s v b$ btd chromosome. Cuticles are depicted anterior up, ventral left. The insert on the lower right hand corner of each panel are enlargements of the sensory structures at the dorsoanterior end of a corresponding cuticle.

Comparing otd expression in wildtype embryos and in embryos with the ectopically expressed btd gene (Fig. 5A,B) confirms the previous observation that head gap genes do not regulate each other's expression (Cohen and Jürgens, 1990; Wimmer et al., 1995; Gao et al., 1996). The ectopic expression of $b t d$ therefore provides an experimental situation where the expression domain of one head gap gene is altered, and the domain of another is kept in its normal spatial limits. To monitor head metamerization in this situation, we used the expression pattern of the segment polarity gene engrailed (en). en expression provides a marker for identifying the number and type of head segments (Fig. 5C; Schmidt-Ott et al., 1994). Expanding the expression domain of btd does not alter the number or shape of the en stripes (Fig. 5D). This indicates that the spatial limits of btd expression are not instructive for metamerization of the head region.

\section{The hunchback>buttonhead transgene provides viable buttonhead function}

The $h b>b t d$ fusion transgene expresses $b t d$ at lower levels than
Fig. 5. Ectopic btd expression does not affect head segmentation. otd protein expression in a wild type blastoderm stage embryo (A) and in an embryo with ectopic $b t d$ expression mediated by the transgene $h b>b t d$ (B). The blue background staining in the yolk of the embryo in $\mathrm{B}$ is due to overstaining for $\beta$-gl expression, in order to identify the genotype of the embryo (see Fig. 3). Note that the otd protein expression is unaltered. (C) At germband extension stage, en protein (brown) is expressed in the ocular (oc), antennal (an), intercalary (ic), mandibular (md) and maxillary (mx) segments. $\beta$-gl (blue) is expressed in the even skipped pattern and indicates a wild type embryo carrying a blue balancer chromosome. (D) The en protein pattern is unchanged in an embryo with ectopic $b t d$ expression mediated by the transgene $h b>b t d$. The genotype of the embryo was identified due to the lack of $\beta$-gl expression.
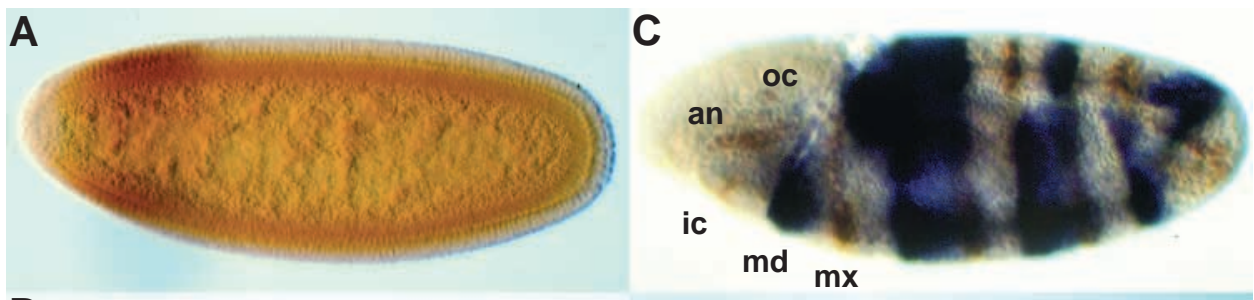

B

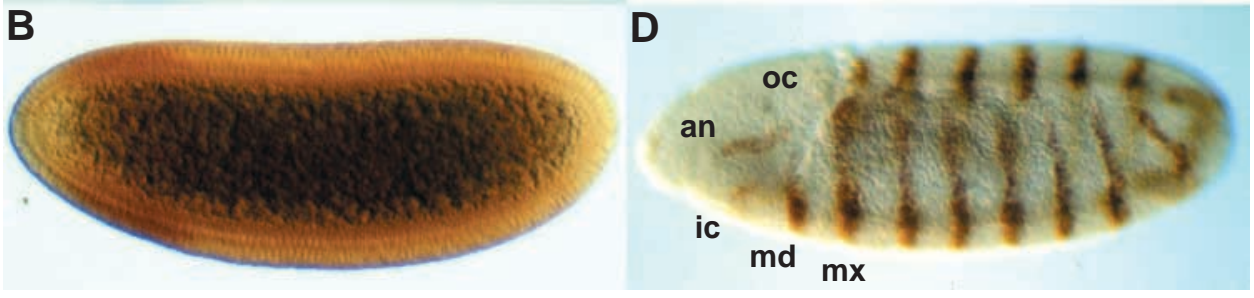


the endogenous gene (Fig. 2I). To rule out that this expression is below the threshold level necessary for btd function, we asked whether it provides sufficient btd activity to rescue a btd null mutation. Using a marked, btd mutant chromosome (Wimmer et al., 1996), we could show that the $h b>b t d$ fusion transgene rescues the embryonic $b t d$ head phenotype (Fig. 4D). Moreover, the fusion transgene rescued males carrying the hemizygous lethal alleles $b t d^{\mathrm{XA}}$ and $b t d^{\mathrm{XG}}$ (Wimmer et al., 1993 ) to adulthood like a wild-type btd transgene (Wimmer et al., 1996). Independent transgenic fly lines which mediate different levels of ectopic transgene expression (Fig. 2G,H) are functionally equivalent. This clearly indicates that the $h b>b t d$ fusion transgene provides sufficient btd activity in the anterior half of the blastoderm embryo to support normal head development.

\section{buttonhead does not contribute to a combinatorial code specifying segment identity}

$b t d$ has been proposed to have a function in head segment specification (Cohen and Jürgens, 1990). otd, ems and btd are each required for the formation of the antennal segment (Fig. 6A; Cohen and Jürgens, 1990). At early blastoderm, btd is normally not expressed in more anterior segments (Fig. 2C,E). Thus, the combination of otd and ems, without btd, has been thought to give rise to the ocular segment (Fig. 6A; Cohen and Jürgens, 1990). According to this model, ectopic expression of btd in the anlage of the ocular segment should change its fate to an antennal one. This should result in the loss of the ocular segment and either in the formation of two adjacent antennal segments or in the fusion of the two segments forming one expanded antennal segment (Fig. 6B).

To examine the effect of ectopic btd expression on segment specification, we analyzed head structures (Jürgens et al., 1986) in cuticle preparations of btd mutant embryos rescued by the $h b>b t d$ fusion transgene (see above). The normal array of the btd dependent structures in the antennal, intercalary and mandibular segments was found (Fig. 4D). Furthermore, those structures which derive from the ocular segment anlage (Schmidt-Ott et al., 1994) and the posterior maxillary segment anlage (Gonzalez-Reyes and Morata, 1991) were normal (Fig. 4D).

Since the ocular segment anlage also gives rise to structures not scoreable in cuticle preparations, we monitored internal sensory structures by the neuronal specific marker mab22C10 (Fujita et al., 1982). btd mutants lack a number of head sensory organs including the dorsal organ (do) and the lateropharygeal organ (lpo) (Fig. 7A,B; Schmidt-Ott et al., 1994). In addition, btd mutant embryos show an abdominal peripheral nervous system (PNS) phenotype (Wimmer et al., 1996), which is not rescued by the $h b>b t d$ fusion transgene. The abdominal btd PNS phenotype was used as an internal marker to identify btd mutant embryos. The presence of the rescued do and lpo indicates that the $h b>b t d$ fusion transgene was fully functional. In such embryos, we analyzed the sensory organs derived from segments adjacent to the btd domain: the Bolwig organ (bo) for the ocular segment and the terminal organ (to) for the posterior part of the maxillary segment. Both organs could be identified and their innervation pattern was normal (Fig. 7C). Thus, the ocular and maxillary segments are specified correctly when btd is ectopically expressed in the anterior half of $b t d$ mutant embryos. This indicates that ectopic
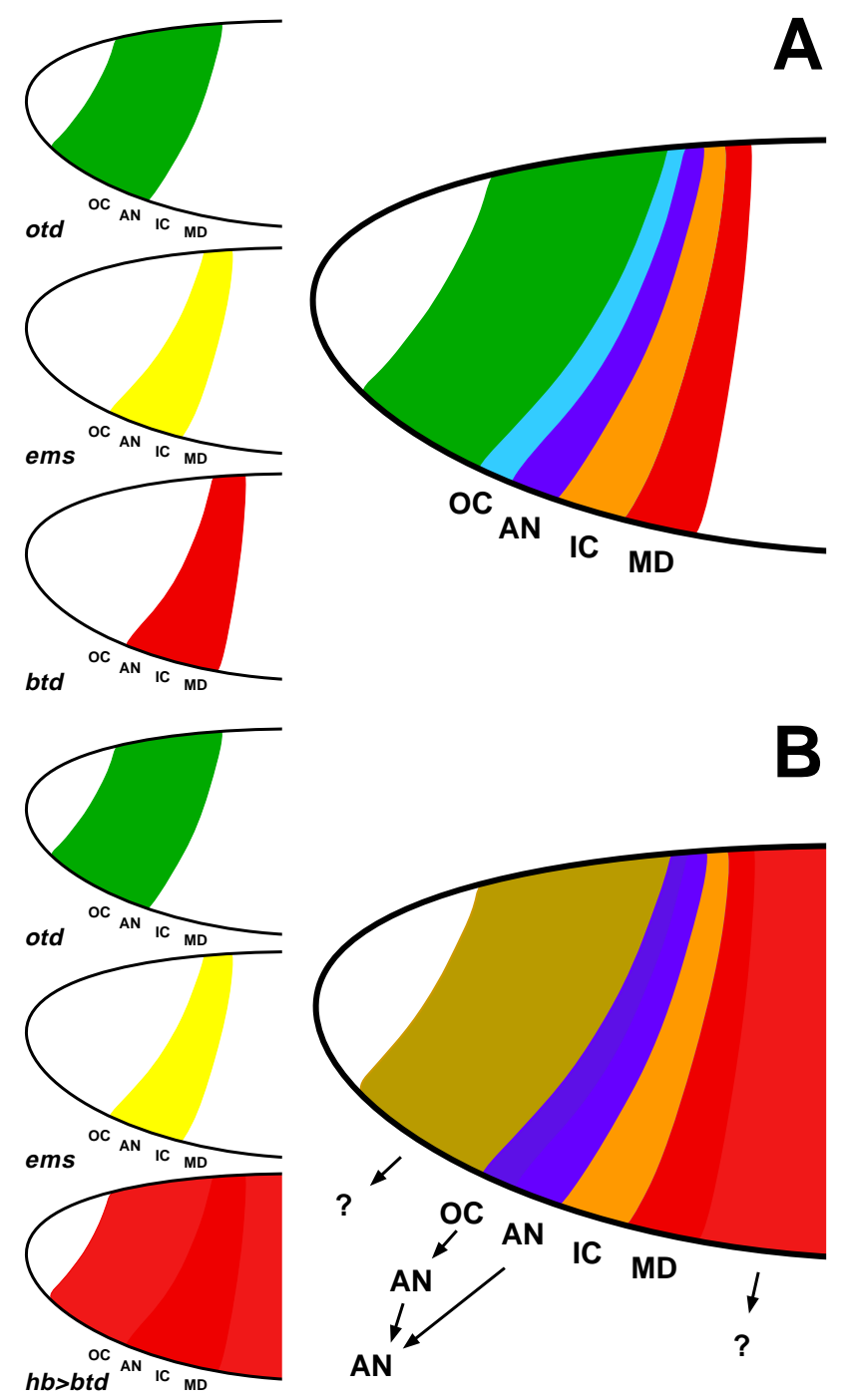

Fig. 6. Schematic representation of the combinatorial model of head segmentation. (A) otd mutants are missing the ocular (OC) and antennal (AN) segments; ems mutants the OC, AN and intercalary (IC) segments; and btd mutants the AN, IC and mandibular (MD) segments (Cohen and Jürgens, 1990; Schmidt-Ott et al., 1994, 1995). The expression patterns of these genes correspond to their mutant phenotypes (see Fig. 2A-E) and it was suggested that the combination otd (green) plus ems (yellow) without btd (red) activity codes for OC (light blue); the combination otd plus ems plus btd for AN (purple); ems plus btd without otd for IC (orange); and btd alone for MD (red) (Cohen and Jürgens, 1990). (B) Ectopic expression of $b t d$ in the anterior half of the embryo (red) changes the proposed code the following way. The code for OC is lost and replaced by the code for AN (purple), which should lead to the formation of two AN or one enlarged AN at the cost of OC. The combinatoral model does not give predictions of what to expect anterior to OC (ochre, ?) and posterior to MD (red, ?).

btd expression covering the anlagen of head and thorax segments does not interfere with specification of segment identity. Fusion transgenes expressing different levels of btd have identical properties (not shown). This observation argues that btd does not contribute to a combinatorial code responsible for specifying head segment identity. 


\section{DISCUSSION}

\section{The IT-system allows for immediate and targeted ectopic gene expression}

We have established an inducible system for region-specific and immediate ectopic gene expression. We developed the ITsystem because the most commonly used ectopic expression systems are not suitable for gene expression in the early blastoderm embryo. The heat shock method (Struhl, 1985) bears several disadvantages: first, the expression cannot be regionally restricted; second, the level of expression varies, is difficult to control and measure, and might exceed physiological levels; third, the heat shock treatment itself can cause phenocopies, especially when applied during the syncitial blastoderm stage, when the gap genes are active (Walter et al., 1990). The GAL4 system was developed to overcome the potential problems of ectopic gene expression causing dominant phenotypes (Brand and Perrimon, 1993). However, it has the disadvantage that it requires two rounds of transcription and translation before the gene of interest is ectopically expressed. This delay in expression excludes the GAL4 system for studying the ectopic expression of gap genes, which represent the first zygotically active genes with a relatively short phenocritical period (Rothe et al., 1992). In contrast, the system presented here allows the coding sequence of interest to be expressed under the direct control of a region-specific promoter, which is temporarily separated by a $f p$-out cassette. After $f p$-out, immediate ectopic expression can occur. We show that the IT-system enables the study of gene functions active very early in development. Moreover, it represents a general tool that controls both position and timing of gene expression, which may be critical for studying other developmental processes such as neurogenesis and organogenesis.

\section{buttonhead encodes a 'generic' transcriptional activator}

We used the IT-system to test the proposed role of the gene $b t d$ in head development. Neither metamerization of the head, nor segment specification are affected when $b t d$ is expressed in regions outside its normal expression domain in blastoderm embryos. This finding indicates that the spatial limits of btd expression are not instructive for head development. The factor encoded by $b t d$ might be a 'generic' transcriptional activator, like its vertebrate homologues Sp1 and Sp4 (Wimmer et al., 1993; Hagen et al., 1995; Supp et al., 1996). As an activator, btd is probably necessary for the expression of several target genes, like cap'n'collar (Mohler, 1993) or collier (Crozatier et al., 1996), whose expression patterns in the head are even more regionally restricted than the btd domain. The expression of these putative btd target genes must therefore be further restricted within the btd domain by other factors. This might explain why ectopic expression of btd outside its normal expression domain has no consequence. The $b t d$ target genes would still be restricted by adjacently acting repressors overriding $b t d$-dependent activation. This, however, raises the question, why btd is normally expressed in a regionally restricted pattern that covers exactly the anlagen of the segments affected in btd mutants (Cohen and Jürgens, 1990; Wimmer et al., 1993, 1996).
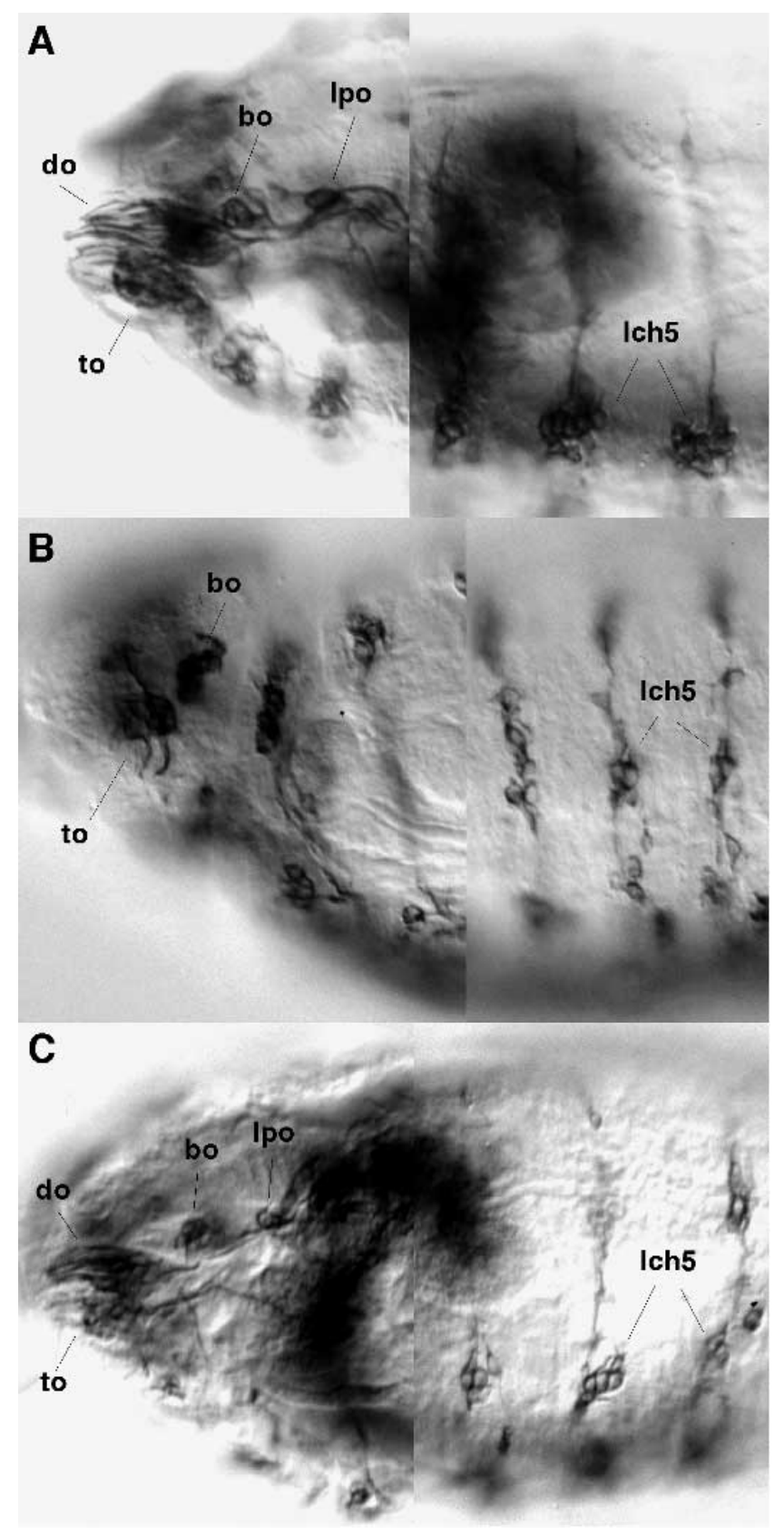

Fig. 7. Ectopic $b t d$ expression does not affect ocular and maxillary segments. (A) Wild-type embryo showing sensory structures detected by mab22C10 (Fujita et al., 1982). (B) btd mutant embryos are missing the dorsal organs (do) and the lateropharyngeal organs (lpo; Schmidt-Ott et al. 1994) and show a reduced number of scolopidia in the lateral pentachordotonal organs (lch5; Wimmer et al., 1996). The terminal organs (to) and the bolwig organs (bo) are present. (C) The $h b>b t d$ fusion transgene rescues the btd mutant head phenotype but not the phenotype in the lch5. Note that the sensory structures derived from the ocular (bo) and maxillary (to) segments are unaltered. Left and right sides of each panel show different focal planes of same embryo.

\section{buttonhead is only required in the early blastoderm}

The $h b>b t d$ fusion transgene does not have any detrimental effects on head development. It shows, however, that $b t d$ expression in the early $h b$ domain is sufficient to rescue $b t d$ 
mutants to adulthood. Since the fusion transgene does not restore btd function in the abdominal PNS (Fig. 7C), the reduced numbers of scolopidia in the chordotonal organs of btd mutants (Wimmer et al., 1996) must still be sufficient for larval survival. Moreover, the proximal $h b$ promoter confers btd expression only in the blastoderm embryo and does not persist through grastulation. Thus, except for its role in the PNS, btd expression seems to be only required in the earliest of its expression domains. This probably reflects the proposed redundant function of $D$-Spl, another $S p 1$ homologue in Drosophila. D-Spl probably substitutes for the lack of btd activity in all the other postblastodermal expression domains of btd (Wimmer et al., 1996).

\section{Head segmentation}

The existence of two different mechanisms that underly head and trunk segmentation, might reflect the evolutionary history of insects. In primitive insects, embryogenesis proceeds in two phases. The head segments are established first, then trunk segments are subsequently added (Sander, 1976). In contrast, segmentation of head and trunk occurs simultanously in Drosophila which may have acquired a special mode of trunk segmentation in order to support the very rapid mode of embryogenesis. The head segmentation mechanisms are likely to be conserved in all insects and even other arthropods (Cohen and Jürgens, 1991), while trunk segmentation appears to have diverged much more extensively (Patel, 1994). Moreover, vertebrate homologs of Drosophila head segmentation genes are expressed during brain development, which suggests an evolutionary conservation of their functions across the animal kingdom (Bally-Cuif and Boncinelli, 1997; Li et al., 1996; Wimmer et al., 1996; and references therein). Our results show that the spatial limits of btd expression are not instructive for head metamerization and raise the question again, of how segmentation in the head region is established. In the anterior head anlage, the blastodermal expression domains of the segment polarity genes wingless and hedgehog each depend specifically on only one head gap gene (Mohler, 1995). Initially these expression domains overlap widely. Due to the mutual exclusiveness of these two genes, interference might generate their segmentally iterated expression pattern and metamerize the anterior head region (Mohler, 1995).

\section{Specifying head segment identity}

The results reported here argue that btd activity does not contribute to the proposed combinatorial code for specification of head segment identity. It might be possible that head segments are not specified by a combinatorial code, but rather by 'phenotypic suppression'. This phenomenon which is based on a hierarchy of gene functions had been noted in the context of Hox gene activities in the trunk (González-Reyes and Morata, 1990). Phenotypic suppression, also referred to as 'posterior prevalence', could explain why ectopic expression of posterior Hox genes overrides the function of more anteriorly expressed Hox genes in both flies and vertebrates (Duboule and Morata, 1994). The molecular explanation for phenotypic suppression proposes competition and differential affinities of the involved homeodomain proteins for similar binding sites (GonzálezReyes et al., 1990). A related phenomenon in opposite direction ('anterior prevalence'), could explain why the ectopic expression of btd in the anterior head region is functionally overridden and therefore of no consequence. However, btd encodes a zinc finger transcription factor and otd and ems code for homeodomain proteins with different DNA binding specificities (Treisman et al., 1992; Wimmer et al., 1993). Thus, if phenotypic suppression exists in the head, it must be mediated by different molecular mechanisms than proposed for the trunk.

The fact that btd is not part of a combinatorial code, does not necessarily exclude a combinatorial model for specifying different head regions. For the gnathal segments, it has been suggested that genes downstream of gap genes control segment identity in a combinatorial manner (Mohler et al., 1995). It is still possible that the homeobox-containing head gap genes otd and ems (Dalton et al, 1989; Finkelstein et al., 1990; Walldorf and Gehring, 1992) provide such a function directly without involving downstream genes. This would imply, however, that after having participated in head metamerization they serve this second function by contributing to a combinatorial code in conjunction with other head genes such as, for example, sloppy paired (Grossniklaus et al., 1994). Our findings clearly show that the zinc finger protein encoded by btd (Wimmer et al., 1993) does not contribute to a combinatorial code that specifies head segments. Thus, btd might act more like the canonical type of gap genes in the trunk, which have no direct role in specifying segment identities (Pankratz and Jäckle, 1993). Therefore, the results presented here contradict the combinatorial model which proposes that the gap-like genes are required in a simultaneous process resulting in head metamerization and specification of head segments (Cohen and Jürgens, 1990).

We thank K. Basler for the original $f p$-out cassette, M. Hoch for the $h b$ promoter, S. Small for pointing out and G. Struhl for providing the $\beta$-tubulin- $f p$ flies, R. Finkelstein for otd antibodies, D. Schmucker for mab22C10 antibodies, A. Goriely for en antibodies, S. Scianimanico for help in generating transgenic fly lines, C. Hartmann and V. Morel for help with stainings, the members of the Jäckle and Desplan laboratories for stimulating discussions and especially $\mathrm{T}$. Turner for all her support. This work was supported by the EMBL (S. M. C.), the Max-Planck Society (H. J.), the Howard Hughes Medical Institute (C. D.), and the Human Frontiers Science Program Organization (H. J., C. D.). E. A. W. was the recipient of an EMBO fellowship.

\section{REFERENCES}

Akam, M. (1987). The molecular basis for metameric pattern in the Drosophila embryo. Development 101, 1-22.

Bally-Cuif, L. and Boncinelli, E. (1997). Transcription factors and head formation invertebrates. BioEssays 19, 127-135.

Brand, A. and Perrimont, N. (1993). Targeted gene expression as a means of altering cell fates and generating dominant phenotypes. Development 118 , 401-415.

Cohen, S. M. and Jürgens, G. (1990). Mediation of Drosophila head development by gap-like segmentation genes. Nature 346, 482-485.

Cohen, S. M. and Jürgens, G. (1991). Drosophila headlines. Trends Genet. 7, 267-272.

Crozatier, M., Valle, D., Dubois, L., Ibnsouda, S. and Vincent, A. (1996). collier, a novel regulator of Drosophila head development, is expressed in a single mitotic domain. Curr. Biol. 6, 707-718.

Dalton, D., Chadwick, R. and McGinnis, W. (1989). Expression and embryonic function of empty spiracles: a Drosophila homeo box gene with two patterning functions on the anterior-posterior axis of the embryo. Gen. Dev. 3, 1940-1956.

Driever, W., Thoma, G. and Nüsslein-Volhard, C. (1989). Determination of spatial domains of zygotic gene expression in the Drosophila embryo by the affinity of binding sites for the bicoid morphogen. Nature 340, 363-367. 
Duboule, D. and Morata, G. (1994). Colinearity and functional hierarchy among genes of homeotic complexes. Trend Genet. 10, 358-364.

Finkelstein, R. and Perrimon, N. (1990). The orthodenticle gene is regulated by bicoid and torso and specifies Drosophila head development. Nature 346 485-488.

Finkelstein, R., Smouse, D., Capaci, T. M., Spradling, A. C. and Perrimon, N. (1990). The orthodenticle gene encodes a novel homeo domain protein involved in the development of the Drosophila nervous system and ocellar visual structures. Genes Dev. 4, 1516-1527.

Fujita, S. C., Zipursky, S. L., Benzer, S., Ferrus, A. and Shotwell, S. L. (1982). Monoclonal antibodies against the Drosophila nervous system. Proc. Natl. Acad. Sci. USA 79, 7929-7933.

Gao, Q., Wang, Y. and Finkelstein, R. (1996). orthodenticle regulation during embryonic head development in Drosophila. Mech. Dev. 56, 3-15.

Gonzalez-Reyes, A. and Morata, G. (1990). The developmental effect of overexpressing a $U b x$ product in Drosophila: embryos is dependent on its interaction with other homeotic products. Cell 61, 515-522.

Gonzalez-Reyes, A., Urquia, N., Gehring, W. J., Struhl, G. and Morata, G. (1990). Are cross-regulatory interactions between homeotic genes functionally significant. Nature $344,78-80$.

Gonzalez-Reyes, A. and Morata, G. (1991). Organization of the Drosophila head as revealed by the ectopic expression of the Ultabithorax product. Development 113, 1459-1471.

Grossniklaus, U., Cadigan, K. M. and Gehring, W. J. (1994). Three maternal coordinate systems cooperate in the patterning of the Drosophila head. Development 120, 3155-3171

Hagen, G., Dennig, J., Preiß, A., Beato, M. and Suske, G. (1995). Functional analyses of the transcription factor Sp4 reveal properties distinct from $\mathrm{Sp} 1$ and Sp3. J. Biol. Chem. 270, 24989-24994

Hartmann, C. and Jäckle. H. (1995). Spatiotemporal relationships between a novel Drosophila stripe expression gene and known segmentation genes by simultaneous visualization of transcript patterns. Chromosoma 104, 84-91.

Ingham, P. W. (1988). The molecular genetics of embryonic pattern formation in Drosophila. Nature 335, 25-34.

Jürgens, G. and Hartenstein, V. (1993). The terminal regions of the body pattern. In The development of Drosophila melanogaster (ed. Bate, M. and Martinez-Arias, A.), pp. 687-746. Cold Spring Harbor Laboratory Press, Cold Spring Harbor, New York.

Jürgens, G., Lehmann, R., Schardin, M. and Nüsslein-Volhard, C. (1986). Segmentation of the head in the embryo of Drosophila melanogaster. Roux's Arch. Dev. Biol. 195, 359-377.

Lardelli, M. and Ish-Horowicz, D. (1993). Drosophila hairy pair-rule gene regulates embryonic patterning outside its apparent stripe domains. Development 118, 255-266.

Laski, F. A., Rio, D. C. and Rubin, G. M. (1986). Tissue specificity of Drosophila $\mathrm{P}$ element transposition is regulated by mRNA splicing. Cell $\mathbf{4 4}$, 7-19.

Li, Y., Brown, S. J., Hausdorf, B., Tautz, D., Denell, R. E. and Finkelstein, R. (1996). Two orthodenticle-related genes in the short-germ beetle Tribolium castaneum. Dev. Genes Evol. 206, 35-45.

Macdonald, P. M. and Struhl, G. (1986). A molecular gradient in early Drosophila embryos and its role in specifying the body pattern. Nature 324, 537-545.

Macdonald, P. M., Ingham, P. W. and Struhl, G. (1986). Isolation, structure and expression of even skipped: a second pair-rule gene of Drosophila containing a homeo box. Cell 47, 721-734.

Martinez-Arias, A. (1993). Development and patterning of the larval epidermis of Drosophila. In The development of Drosophila melanogaster (ed. Bate, M. and Martinez-Arias, A.), pp. 467-516. Cold Spring Harbor Laboratory Press, Cold Spring Harbor, New York

McGinnis, W. and Krumlauf, R. (1992). Homeobox genes and axial patterning. Cell 68, 283-302.

Michiels, F., Gasch, A., Kaltschmidt, B. and Renkawitz-Pohl, R. (1989). A 14 bp promoter element directs the testis specificity of the Drosophila $\beta$ tubulin gene. EMBO J. 8, 1559-1565.

Mohler, J. (1993). Genetic regulation of CNC expression in the pharyngeal primordia of Drosophila blastoderm embryos. Roux's Arch. Dev. Biol. 202, 214-223.

Mohler, J. (1995). Spatial regulation of segment polarity gene expression in the anterior terminal region of the Drosophila blastoderm embryo. Mech. Dev. 50, $151-161$

Mohler, J., Mahaffey, J. W., Deutsch, E. and Vani, K. (1995). Control of Drosophila head segment identity by the bZIP homeotic gene cnc. Development 121, 237-247.
Nüsslein-Volhard, C. and Wieschaus, E. (1980). Mutations affecting segment number and polarity in Drosophila. Nature 287, 795-801.

Pankratz, M. J. and Jäckle, H. (1993). Blastoderm segmentation. In The development of Drosophila melanogaster (ed. Bate, M. and Martinez-Arias, A.), pp. 467-516. Cold Spring Harbor Laboratory Press, Cold Spring Harbor, New York.

Parkhurst, S. M. and Ish-Horowicz, D. (1991). Mis-regulating segmentation gene expression in Drosophila. Development 111, 1121-1135.

Patel, N. H., Martin-Blanco, E., Coleman, K. G., Poole, S.J., Ellis, M. C., Kornberg, T. B. and Goodman, C. S. (1989). Expression of engrailed proteins in arthropods, annelids, and chordates. Cell 58, 955-968.

Patel, N. H. (1994). The evolution of arthropod segmentation: insights from comparision of gene expression patterns. Development Supplement, 201207.

Pirrotta, V. (1988). Vectors for P-element transformation in Drosophila. In Vectors. A survey of molecular cloning vectors and their uses (eds. Rodriguez, R.L. and Denhardt, D.T.), pp. 437-456. Butterworths, Boston and London.

Rothe, M., Pehl, M., Taubert, H. and Jäckle, H. (1992). Loss of gene function through rapid mitotic cycles in the Drosophila embryo. Nature 359, 156-159.

Rubin, G. M. and Spradling, A. C. (1982). Genetic Transformation of Drosophila with transposable element vectors. Science 218, 348-353.

Sander, K. (1976). Specification of the basic body pattern in insect embryogenesis. Adv. Insect Physiol. 12, 125-238.

Schmidt-Ott, U., González-Gaitán, M., Jäckle, H. and Technau, G. M. (1994). Number, identity and sequence of the Drosophila head segments as revealed by neural elements and their deletion patterns in mutants. Proc. Natl. Acad. Sci. USA 91, 8363-8367.

Schmidt-Ott, U., González-Gaitán, M. and Technau, G. M. (1995). Analysis of neural elements in head-mutant Drosophila embryos suggests segmental origin of the optic lobes. Roux's Arch. Dev. Biol. 205, 31-44.

Schröder, C., Tautz, D., Seifert, E. and Jäckle, H. (1988). Different regulation of the two transcripts from the Drosophila gap segmentation gene hunchback. EMBO J. 7, 2881-2887.

St Johnston, D. and Nüsslein-Volhard, C. (1992). The origin of pattern and polarity in the Drosophila embryo. Cell 68, 201-219.

Struhl, G. (1985). Near reciprocal phenotypes caused by inactivation or indiscriminate expression of the Drosophila segmentation gene $f t z$. Nature 318, 677-680.

Struhl, G., Struhl, K. and Macdonald, P. M. (1989). The gradient morphogen bicoid is a concentration-dependent transcriptional activator. Cell 57, 12591273 .

Struhl, G. and Basler, K. (1993). Organizing activity of wingless protein in Drosophila. Cell 72, 527-540.

Struhl, G., Fitzgerald, K. and Greenwald, I. (1993). Intrinsic activity of the Lin-12 and Notch intracellular domains in vivo. Cell 74, 331-345.

Supp, D. M., Witte, D. P., Branford, W. W., Smith, E. P. and Potter, S. S. (1996). Sp4, a member of the Sp1-family of zinc finger transcription factors, is required for normal murine growth, viability and male fertility. Dev. Biol. 176, 284-299.

Treisman, J., Harris, E., Wilson, D. and Desplan, C. (1992). The homeodomain: a new face for the helix-turn-helix? Bioessays 14, 145-150.

Vazquez, J. and Schedl, P. (1994). Sequences required for enhancer blocking activity of scs are located within two nuclase-hypersensitive regions. $E M B O$ J. 13, 5984-5993

Walldorf, U. and Gehring, W. J. (1992). empty spiracles, a gap gene containing a homeobox involved in Drosophila head development. EMBO J. 11, 2247-2259

Walter, M. F. Petersen, N. S. and Biessmann, H. (1990). Heat shock causes the collapse of the intermediate filament cytoskeleton in Drosophila embryos. Dev. Gen. 11, 270-279.

Wieschaus, E., Perrimon, N. and Finkelstein, R. (1992). orthodenticle activity is required for the development of medial structures in the larval and adult epidermis of Drosophila. Development 115, 801-811.

Wimmer, E. A., Jäckle, H., Pfeifle, C. and Cohen, S. M. (1993). A Drosophila homologue of human $\mathrm{Sp} 1$ is a head-specific segmentation gene. Nature 366, 690-694

Wimmer, E. A., Simpson-Brose, M., Cohen, S. M., Desplan, C. and Jäckle, H. (1995). Trans- and cis-acting requirements for blastodermal expression of the head gap gene buttonhead. Mech. Dev. 53, 235-245.

Wimmer, E. A., Frommer, G., Purnell, A. P., and Jäckle, H. (1996) buttonhead and D-Sp1: a novel Drosophila gene pair. Mech. Dev. 59, 53-62.

(Accepted 30 January 1997) 